\title{
Investigating the impact of self-management behaviours on quality of life and fear of recurrence in head and neck cancer survivors: A population-based survey
}

\author{
Simon Dunne ${ }^{1}$ (D) । Laura Coffey ${ }^{2}$ | Linda Sharp ${ }^{3}$ | Deirdre Desmond ${ }^{2}$ | Claire Cullen ${ }^{1}$ | \\ Jean O'Connor ${ }^{4}$ | Eleanor O'Sullivan ${ }^{5}$ | Conrad Timon ${ }^{6}$ | Pamela Gallagher ${ }^{1}$
}

${ }^{1}$ School of Nursing and Human Sciences, Dublin City University, Dublin, Ireland

${ }^{2}$ Department of Psychology, Maynooth University, County Kildare, Ireland

${ }^{3}$ Institute of Health and Society, Newcastle University, Newcastle, UK

${ }^{4}$ National Cancer Registry Ireland, Cork, Ireland

${ }^{5}$ Dental School and Hospital, University College Cork, Cork, Ireland

${ }^{6}$ Head and Neck Cancer Programme, St. James's Hospital, Dublin, Ireland

\section{Correspondence}

Pamela Gallagher, School of Nursing and Human Sciences, Dublin City University, Dublin, Ireland.

Email: pamela.gallagher@dcu.ie

Funding information

Irish Cancer Society and the Health Research Board, Grant/Award Number: SRP13GAL, MRCG/2013/11

\begin{abstract}
Objectives: Emerging cancer-survivorship research suggests that self-management can lead to improved outcomes. However, research examining the impact of self-management behaviours on quality of life (QoL) and fear of recurrence (FoR) in cancer survivors is lacking. This study investigated the relationship between self-management behaviours and QoL and FoR following treatment for head and neck cancer (HNC).
\end{abstract}

Methods: Postal surveys were sent to 734 eligible HNC survivors (ICD10 C01-C14; C32) in the Republic of Ireland who were 12- to 60-months post diagnosis. QoL and FoR were measured using the Functional Assessment of Cancer Therapy (FACT-G and Head and Neck Cancer Subscale) measure and Fear of Relapse/Recurrence Scale, respectively. Seven self-management behaviours were measured using the Health Education Impact Questionnaire.

Results: Three hundred and ninety-five HNC survivors completed surveys $150.3 \%$ response rate). After controlling for sociodemographic and clinical characteristics, self-management behaviours accounted for $20 \%$ to $39.4 \%$ of the variance in QoL and FoR. Higher scores on positive and active engagement in life, constructive attitudes and approaches, and skill and technique acquisition were significantly associated with higher global QoL and lower FoR, whilst higher scores on positive and active engagement in life and constructive attitudes and approaches only were significantly associated with higher HNC-specific QoL. Additionally, lower scores on self-monitoring and insight were significantly associated with higher HNC-specific and global QoL and lower FoR.

Conclusions: The findings highlight the potential utility of self-management interventions promoting active problem solving, positive self-talk, and skill acquisition amongst cancer survivors. However, increased self-monitoring may relate to negative outcomes in HNC, a finding that warrants further investigation.

\section{KEYWORDS}

cancer, cancer survivorship, fear of recurrence, head and neck cancer, oncology, psycho-oncology, quality of life, self-management 


\section{1 | INTRODUCTION}

Medical advances in recent decades have increased the number of people who are living longer with cancer and its consequences. ${ }^{1}$ Many cancer survivors' daily lives are affected by ongoing physical, emotional and social needs arising from their condition and its treatment. ${ }^{2}$ Arising from the enduring nature of these needs, survivors must respond to new and evolving demands and assume an active role in maintaining health and well-being following primary treatment. ${ }^{3}$ In this context, cancer selfmanagement has been defined as "awareness and active participation by the person in their recovery, recuperation and rehabilitation, to minimise the consequences of treatment, and promote survival, health and well-being" (p. 6). ${ }^{4}$ Key self-management behaviours in cancer survivorship include health-directed behaviours like eating a healthy diet, ${ }^{5}$ monitoring the consequences of cancer and its treatment, ${ }^{3}$ effectively navigating health-services resources, ${ }^{6}$ adopting constructive attitudes to manage emotional challenges, ${ }^{7}$ and drawing upon social support. ${ }^{8}$ Emerging research suggests that interventions, which promote such behaviours in cancer survivorship, can "improve symptoms of fatigue, pain, depression, anxiety, emotional distress and quality of life (QoL)" (p.1323). ${ }^{9}$

Head- and neck-cancer (HNC) survivors often have wide-ranging needs requiring ongoing self-management. These include eating and speech difficulties, facial disfigurement, and elevated distress, which have marked negative effects on survivors' QoL following treatment. ${ }^{10-12}$ Fear of recurrence (FoR) can also be a persistent and significant issue in this population, with noted deleterious effects on QoL ${ }^{12,13}$; FoR is the most frequently cited concern by HNC survivors aged less than 70 years old. ${ }^{14}$ Our recent qualitative research has illustrated the variety of strategies that HNC survivors use to self-manage these ongoing issues, ${ }^{15}$ the barriers they encounter in their self-management attempts, ${ }^{16}$ and the processes through which they integrate selfmanagement into their daily lives. ${ }^{17}$ However, to our knowledge, neither there has been no research on the relationship between selfmanagement behaviours and QoL in this population to date nor has there been any research on the relationship between self-management behaviours and FoR in cancer more generally. Understanding the relationship between self-management and these key outcomes in cancer survivorship is important in order to inform the content and delivery of self-management interventions designed to develop the skills and confidence that cancer survivors need to live well following treatment. The aim of the current paper was to examine the degree to which HNC survivors' performance of self-management behaviours were associated with their QoL and FoR in the post-treatment period, after controlling for key demographic and clinical characteristics. We hypothesised that self-management behaviours would be associated with higher QoL and lower FoR amongst HNC survivors.

\section{2 | MATERIALS AND METHODS}

\section{1 | Design, participants, and recruitment procedures}

We adopted a national population-based postal-survey design targeting HNC survivors in the Republic of Ireland. This population-based survey is part of a larger study to develop a selfmanagement intervention to improve quality of life amongst HNC survivors. We received ethical approval from 20 hospitals across the country, ${ }^{1}$ including all major treatment centres for HNC. Between May to June 2015, we identified 1208 individuals eligible for the study in collaboration with the National Cancer Registry Ireland (NCRI), which records all new primary cancers diagnosed amongst people residing in the country. The study population was identified from the NCRI using the following criteria to indicate that an individual (a) was alive; (b) was aged 18 years old or older at diagnosis; (c) had obtained a diagnosis of a primary invasive tumour in the head and neck region, including cancers of the oral cavity, oropharynx, larynx, hypopharynx, paranasal sinuses, and salivary glands (ICD10 C01-C14, C32); (d) was 1 to 5 years post diagnosis (to ensure post-treatment status); (e) had an absence of a second invasive cancer diagnosed after HNC (excepting nonmelanoma skin cancer); and (f) had been treated in one of the 20 participating hospitals. We then identified 82 consultants who had treated these survivors and requested their assistance to screen them to confirm eligibility. Twenty-seven consultants, accounting for $125 \mathrm{HNC}$ survivors, either did not agree or failed to respond to our screening request. The remaining 55 treating consultants (with 1083 eligible patients) agreed to screen a list of their patients and confirm eligibility. These consultants confirmed that an individual (a) was alive, (b) was aware they had cancer, (c) was not receiving palliative care, (d) had not developed a second invasive cancer (apart from nonmelanoma skin cancer), (e) had completed primary treatment for HNC, (f) was not receiving treatment for a recurrence or secondary cancer, (g) was considered disease-free for the preceding 4 months, and (h) there was no medical or other reason why it would be inappropriate to contact them about the study. Following this screening process, survey packs including a survey booklet, explanatory letter, information sheet, consent form, and stampedaddressed return envelope were posted to 785 eligible individuals between November 2015 to March 2016. Nonresponders received up to two reminder letters (at 2-4 weeks after issue of the survey pack). Figure S1 further illustrates the screening/recruitment process for interested readers.

Participants were asked to indicate if they received help filling in the questionnaires (eg, because of poor sight, and difficulty writing). The survey, which we anticipated would take approximately 30 minutes to complete, contained ten sections; only data pertaining to demographics, self-management behaviours, QoL, and FoR are reported in the present paper.

${ }^{1}$ Ethical approval/letter dates: Beaumont_Hospital_REC_No.15/38; Cork University_Hospitals_ECM_4_(aa)_06/05/14/ ECM_3_(y)_07/07/15; Galway_ University_Hospitals_C_A_1195; University_Hospital_Limerick_REC_Approval 17/04/15; Research_Ethics_Committee_HSE-Midland Area_Ref_010415PG; University_Hospital_Waterford_REC_Approval_10/05/15; Letterkenny_Hospitals_REC_Approval_22/04/15; Mater_Misercordiae_University_Hospital_REC _Ref_1/378/1711; RVEEH_REC_Approval_23/02/15; SJH/AMNCH_REC_ 2015-02_Chairman's_Action_(16); Sligo_Regional_Hospital_REC_Approval 09/03/15; St.Vincent's_Healthcare_Group_Limited_Ethics_and_Medical_ Research_Committee_11/07/15. 


\section{2 | Measures}

We used the Health Education Impact Questionnaire (HEIQ) to determine participants' use of self-management behaviours. There is currently an absence of high-quality instruments to measure selfmanagement behaviours. However, this measure has been extensively used for this purpose across a broad range of chronic conditions and was devised by Australian researchers to assess the efficacy of patient education programs in teaching key self-management behaviours. ${ }^{18}$ The measure asks respondents to indicate their level of agreement with a series of statements across eight domains (42 items) on four-point Likert scales and has been used as a measure of self-management behaviours with other groups of cancer patients. ${ }^{19,20}$ Higher scores in seven domains indicate greater performance of specific selfmanagement behaviours (positive and active engagement in life, health-directed behaviour, constructive attitudes and approaches, skill and technique acquisition, self-monitoring and insight, health-service navigation, and social integration and support). Higher scores on the emotional well-being domain denote higher levels of negative affect.

We assessed QoL using the Functional Assessment of Cancer Therapy (FACT-G). This consists of 27 statements, which ask participants to consider the previous week and subsequently rate their QoL across physical, social/family, emotional, and functional domains on a five-point scale. We also used the FACT HNC-specific domain, the Head and Neck Cancer Scale (HNCS). This comprises 12 statements about HNC-specific challenges and can be used independently of the FACT-G to indicate HNC-specific QoL. FACT-G and HNCS scores were computed for all individuals based on the authors' scoring instructions. In all cases, higher domain scores indicate higher QoL. The FACT-G and HNCS have been widely used and validated in different countries ${ }^{21,22}$ and are commonly used to assess the QoL of HNC survivors. ${ }^{12}$

We assessed FoR using the Fear of Relapse/Recurrence Scale (FRRS). ${ }^{23}$ On this measure, respondents are asked to rate five statements on a five-point Likert scale as they apply to the previous week. Scores range from 5 to 25 with higher scores indicative of higher levels of FoR. Thewes et $\mathrm{al}^{24}$ have found that this scale is the most extensively validated brief measure of FoR with desirable psychometric properties.

Sociodemographic and clinical data was collected via NCRI data and survey data. We obtained NCRI data on participants' age (in years) at time of survey completion, sex, cancer site (four variables categorised as larynx, salivary glands, oral cavity, or pharynx, respectively), treatment received within the first year since diagnosis (three variables categorised as whether not the individual had received cancer-directed surgery, radiotherapy, or chemotherapy), and cancer stage at diagnosis. Survey data were used to indicate participants smoking status (current smoker or nonsmoker), relationship status (in a relationship or not), education level (primary, secondary, third level/postgraduate, or unknown), presence of a comorbidity (at least one or none), and alcohol use (extent of use, assessed using the Alcohol Use Disorders Identification Test [AUDIT-C], ${ }^{25}$ which measures the extent of problem drinking behaviours across three items; scores above four indicate that an individual's drinking may be negatively affecting their safety).

\section{3 | Statistical methods}

In the following analyses, we only included the seven domains of the HEIQ relating to self-management behaviours (ie, we excluded emotional well-being as it is a measure of negative affect). Cronbach's alpha values for all included scales and associated subscales were between 0.83 and 0.94. For respondent/nonrespondent analysis, we used logistic regression with key sociodemographic and clinical characteristics aggregated from survey and NCRI data as predictor variables. We used hierarchical multiple regression analysis to explore associations between self-management behaviours and global QoL, HNC-specific QoL, and FoR scores, respectively, after controlling for relevant sociodemographic and clinical characteristics.

In preparation for hierarchical multiple regression analyses, we explored the relationships between key sociodemographic and clinical characteristics (age at diagnosis, sex, self-reported current smoking status, extent of alcohol use, presence of a comorbidity, treatment received, cancer location, cancer stage at diagnosis, employment status, relationship status, number of months between diagnosis and survey completion, and whether or not a respondent was helped to complete the survey) and outcome variables through relevant tests of associations (eg, correlations, $\mathrm{t}$ tests, and Chi-squared test). This exploratory analysis revealed that age at diagnosis, current smoking status, extent of alcohol use, and comorbidity presence had significant relationships with global QoL and FoR and were retained as covariates in multivariate analyses. These same four variables, in addition to three binary predictors relating to cancer type (larynx, salivary glands, and pharynx), also had significant relationships with HNC-specific QoL; all seven were retained as covariates for multivariate analysis. In each model, we assessed the independent effects of self-management behaviours on our outcomes by entering pertinent sociodemographic and clinical covariates in the first block and self-management behaviours in the second block. We made the a priori decision to exclude the HEIQ subscale social integration and support from global QoL multivariate analyses due to potential multi-collinearity with the Social Well-Being subscale, which contributes to FACT-G scores. Although there are subtle differences between these two subscales in how they deal with social interactions (the former specifically relates to receiving help for one's health-related issues, whilst the latter relates to one's sense of social well-being more generally), we were concerned that the inclusion of the social integration and support subscale would inflate the error variance in our analysis of global QoL.

All tests were two-tailed with an alpha level set at 0.05 . All statistical analyses were performed using IBM Statistics Package for the Social Sciences, Version 23.

\section{3 | RESULTS}

Sociodemographic and clinical characteristics for participants are provided in Table 1. Of the 785 individuals invited to participate, 395 
TABLE 1 Sociodemographic/clinical characteristics of participants

\begin{tabular}{|c|c|c|c|c|c|}
\hline Variable & $\mathrm{N}$ & Variable & $\mathrm{N}$ & Variable & $N$ \\
\hline Total & 395 & & & & \\
\hline Sex & & Time since diagnosis & & Self-reported smoking status & \\
\hline Female & 123 & $25-36 \mathrm{mo}$ & 98 & Ex-smoker & 221 \\
\hline Relationship status & & $37-48 \mathrm{mo}$ & 95 & Never smoked & 90 \\
\hline Married/cohabiting & 248 & $49-60 \mathrm{mo}$ & 77 & Unknown & 15 \\
\hline Unknown & 15 & Larynx & 119 & $25-39$ years & 18 \\
\hline Treatment(s) & & Salivary glands & 24 & $40-54$ years & 104 \\
\hline Chemotherapy only & 4 & Oral cavity & 143 & $55-69$ years & 197 \\
\hline Radiotherapy only & 64 & Pharynx & 110 & 70 years or more & 76 \\
\hline Surgery/radiotherapy & 64 & III & 44 & Third level/Postgraduate & 89 \\
\hline Surgery/radiotherapy/chemotherapy & 30 & IV & 103 & Unknown & 5 \\
\hline Unknown & 68 & Unknown & 90 & & \\
\hline Employment status & & Presence of a comorbidity & & & \\
\hline Currently working & 110 & Yes & 196 & & \\
\hline Not working/Retired & 280 & No & 196 & & \\
\hline Unknown & 5 & Unknown & 3 & & \\
\hline
\end{tabular}

completed and returned surveys (50.32\% response rate). Ninety-five participants $(24.1 \%)$ required help in completing the survey. Logistic regression analysis revealed no significant differences between respondents and nonrespondents on the following characteristics (aggregated from survey and NCRI data): age at diagnosis, sex, current smoking status, treatment received, cancer type, cancer stage, or time since diagnosis.

Prior to multivariate analyses, preliminary analyses confirmed that the data did not violate any assumptions of multiple regression. Relevant tests of association for all variables included in the analyses are provided in Table S1. All seven self-management behaviours were correlated with each other to a highly significant degree $(P<0.001$ in each case).

When we entered sociodemographic and clinical covariates (age at diagnosis, self-reported current smoking status, extent of alcohol use, and presence of a comorbidity) in the first block of our global QoL model $(M=80.81, S D=18.58)$, they accounted for $11.7 \%$ of the variance $\left(F_{4,306}=10.17, P<0.001\right)$. The six self-management behaviours entered in block 2 accounted for an additional $39.4 \%$ of variance in QoL; $R^{2}$-change $=0.394, F$-change $_{6,300}=40.27$, $P<0.001$. The final model accounted for $51.1 \%$ of variance in QoL scores; $F_{10,300}=31.36, P<0.001$. The following self-management variables independently predicted global QoL: positive and active engagement in life, self-monitoring and insight, constructive attitudes and approaches, and skill and technique acquisition. Of these, positive and active engagement in life $(B=17.27 ; \mathrm{Cl}, 12.67-21.87 ; P<0.001)$ and constructive attitudes and approaches $(B=9.84 ; \mathrm{Cl}, 5.85-13.82$;
$P<0.001)$ had the strongest positive association with global QoL, whilst self-monitoring and insight was the only self-management behaviour to have a negative association with global $\mathrm{QoL}(B=-9.23 ; \mathrm{Cl}$, 15.13 to 3.33; $P<0.01$ ).

For our HNC-specific QoL ( $M=27.76, S D=8.46$ ) model, the sociodemographic and clinical covariates accounted for $15.1 \%$ of variance in HNC-specific QoL scores $\left(F_{7,303}=7.71, P<0.001\right)$. The seven self-management behaviours entered in the second block of this model explained an additional $20 \%$ of variance; $R^{2}$-change $=0.20$, $F$-change $_{7,296}=13.01, P<0.001$. The final model accounted for $35.1 \%$ of the variance in HNC-specific QoL; $F_{14,296}=11.43$, $P<0.001$. The following self-management variables independently predicted HNC-specific QoL: positive and active engagement in life, self-monitoring and insight, and constructive attitudes and approaches. Positive and active engagement in life had the strongest positive impact on HNC-specific QoL ( $B=5.73 ; \mathrm{Cl}, 3.26-8.20 ; P<0.001$ ); self-monitoring and insight was the only self-management behaviour to have a negative relationship with $\mathrm{HNC}$-specific $\mathrm{QoL}(\mathrm{B}=-5.17 ; \mathrm{Cl},-8.32$ to 2.01; $P<0.01$ ).

In relation to FoR $(M=13.74, S D=4.57)$, block 1 variables accounted for $7.2 \%$ of variance $\left(F_{4,306}=5.94, P<0.001\right)$. The seven self-management behaviours accounted for an additional $32.9 \%$ of variance; $R^{2}$-change $=0.329, F$-change ${ }_{7,299}=23.49, P<0.001$. The final model accounted for $40.1 \%$ of variance in FoR; $F_{11,299}=18.22$, $P<0.001$. The following self-management variables independently predicted FoR: positive and active engagement in life, self-monitoring and insight, constructive attitudes and approaches, and skill and 


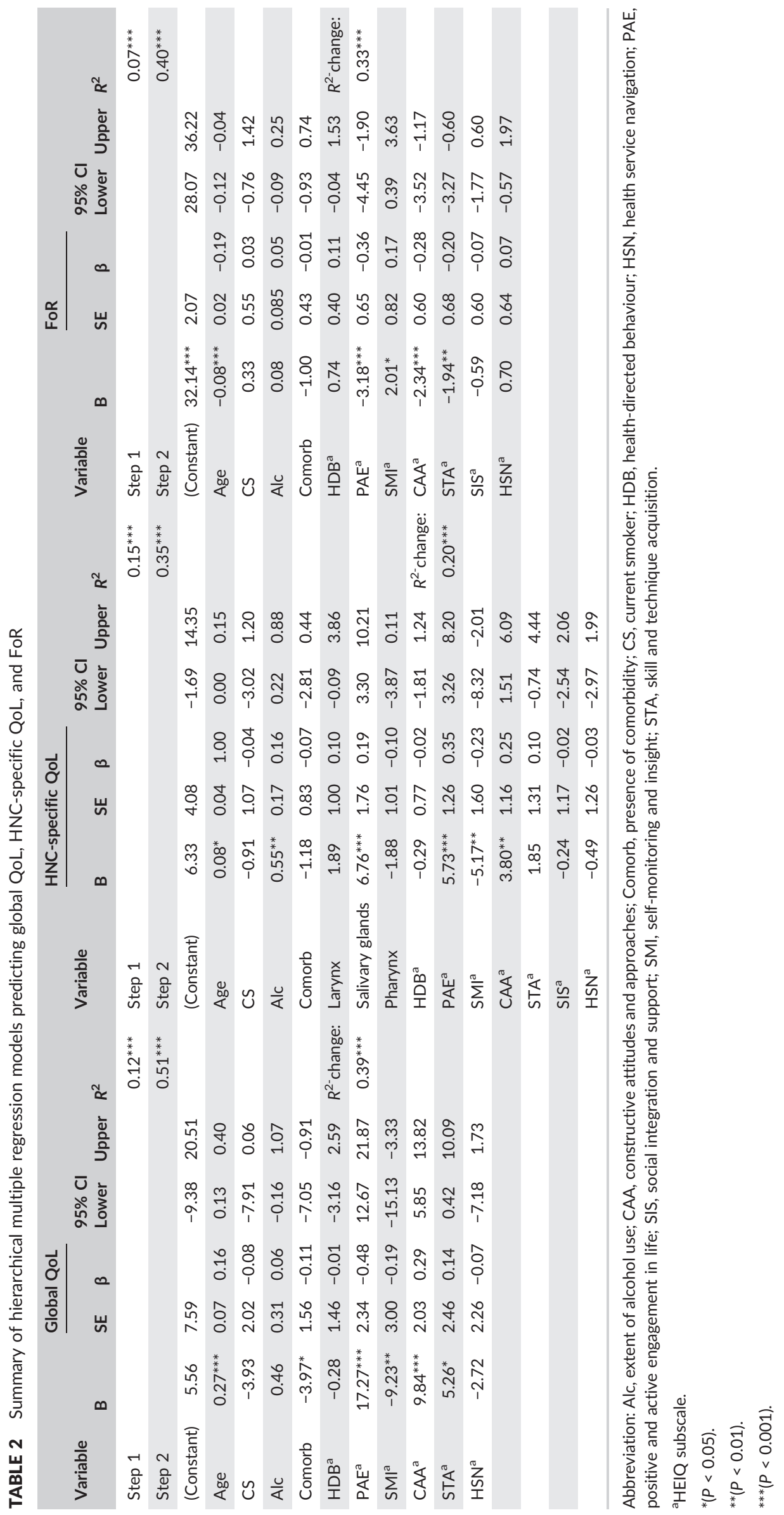


technique acquisition. Positive and active engagement in life $(B=-3.18$; $\mathrm{Cl},-4.45$ to $1.90 ; P<0.001)$ and constructive attitudes and approaches $(B=-2.34 ; C l,-3.52$ to $1.17 ; P<0.001)$ were most strongly associated with lower FoR, whilst self-monitoring and insight was the only selfmanagement behaviour to be associated with increased FoR $(B=2.01 ; \mathrm{Cl}, 0.39-3.63 ; P<0.05)$ amongst participants. Further details on the models are provided in Table 2.

\section{4 | CONCLUSIONS}

To our knowledge, this is the first study to investigate the relationship between key self-management behaviours and QoL and FoR in HNC survivors and the first to investigate the relationship between selfmanagement behaviours and FoR in cancer more generally. Our results demonstrate that higher scores on three key self-management behaviours (positive and active engagement in life, constructive attitudes and approaches, and skill and technique acquisition) were significantly associated with higher global QoL and lower FoR amongst HNC survivors who had completed their active treatment. In contrast, higher self-monitoring and insight were significantly associated with lower levels of QoL and higher FoR in our sample. The global QoL findings were also replicated with HNC-specific QoL, with the exception of a nonsignificant association between skill and technique acquisition and HNC-specific QoL.

Our findings that positive and active engagement in life, constructive attitudes and approaches, and skill and technique acquisition were significantly associated with increases in global QoL and decreases in FoR and that positive and active engagement in life and constructive attitudes and approaches were associated with increases in HNC-specific QoL are consistent with the wider cancer self-management literature. For instance, positive appraisals such as optimism, hope, and inner strength have been linked to positive outcomes such as benefit finding, post-traumatic growth, and higher QoL in individuals with $\mathrm{HNC}^{7,26}$ and greater psychosocial adjustment and lower perceived stress amongst cancer survivors more generally. ${ }^{27,28}$ Engaging in active problem solving is a core component of cancer selfmanagement interventions, ${ }^{29}$ whilst acquiring new skills and techniques (eg, relating to medical management of HNC site) is recognised as critical in HNC rehabilitation. ${ }^{30}$ Importantly, our findings indicate that these self-management behaviours are strongly associated with improved QoL and reduced FoR amongst HNC survivors, even after controlling for pertinent sociodemographic and clinical factors. In line with these findings, HNC survivors might benefit from structured self-management interventions with components that focus on active problem solving, positive appraisal, and acquiring specific skills and techniques that are relevant to self-managing their condition (whilst a thorough discussion of such interventions is beyond the scope of the current paper, interested readers are directed to the review by Davies \& Batehup ${ }^{4}$ on this topic).

An additional striking finding in this study was that increased levels of self-monitoring and insight were associated with poorer QoL and FoR outcomes in HNC survivors. Whilst these findings were contrary to our hypotheses relating to self-monitoring, they are perhaps unsurprising, given that elevated levels of self-monitoring may be closely linked to hypervigilance to signs of recurrence in cancer survivors. Hypervigilance is a key component in conceptualisations of FoR ${ }^{31}$ and maladaptive coping in $\mathrm{HNC}^{32,33}$ and is associated with the incidence of post-traumatic stress disorder in cancer survivorship $^{34,35}$ In contrast, self-monitoring has typically been regarded as adaptive for individuals with cancer, ${ }^{3}$ with recommendations to target this behaviour in cancer self-management interventions ${ }^{9}$ and qualitative accounts supporting its positive effects. ${ }^{36}$ In light of this, further research is needed to gain a better understanding of the impact of self-monitoring on outcomes in HNC survivorship, eg, to establish if a threshold point exists where adaptive self-monitoring becomes maladaptive hypervigilance in HNC survivors. Furthermore, selfmanagement behaviour scales like the HEIQ may need to be revised for use in cancer survivors generally in order to account for the potential continuum of adaptive self-monitoring to maladaptive hypervigilance in cancer survivorship.

We also found that higher scores on health-directed behaviours, health service navigation, and social integration and support were associated with higher QoL and lower FoR amongst HNC survivors in univariate analyses, but these associations did not remain significant after controlling for relevant sociodemographic and clinical characteristics. This suggests that the variance in QoL and FoR attributable to these self-management behaviours may have been accounted for by our covariates or may have been confounded by the presence of other self-management behaviours with which they were highly correlated. In spite of this, extant research has demonstrated the importance of these behaviours in cancer self-management. ${ }^{5,6,8}$ With this in mind, further research is needed to determine the extent to which these behaviours benefit cancer survivors across various stages of the cancer trajectory and/or in relation to other important outcomes (eg, 5-year survival). Furthermore, because of their potential shared variance with other variables, more refined measures of these selfmanagement behaviours may be required, which better capture the potential unique variance that they account for in relation to important outcomes for HNC survivors (and, potentially, in relation to other cancer types). Indeed, the work of Schuler et $\mathrm{al}^{37}$ has indicated that this instrument may need to be adapted for specific conditions like HNC.

\section{1 | Study limitations}

The current study was cross-sectional; as such, we cannot be certain of the direction of associations between self-management behaviours, QoL and FoR. Future longitudinal research is needed to establish this and whether or not there may be different relationships between HNC survivors' use of self-management behaviours and their QoL and FoR at different stages in the post-treatment trajectory. Our sample also comprised HNC survivors with a wide range of time since the end of their treatment. Emerging research suggests there are core differences between HPV- and non-HPV-related HNC survivors in their $\mathrm{QoL}^{38}$; NCRI data relating to HPV was not available to us for 
HNC cancer survivors at the time of data collection, so we could not explore this. Additionally, 27 consultants (accounting for approximately $10 \%$ of HNC survivors meeting our eligibility criteria in Ireland) did not take part in our study. We were also unable to adjust for consultants or treating hospitals in our analyses as the NCRI data available to us was limited to the physician and hospital site registered at diagnosis (and these may not be the centre/clinician responsible for the survivor's cancer care); these variables may have accounted for some of our unexplained variance. Nonetheless, the rigorous populationbased survey design was a clear strength, ensuring the sample was broadly reflective of HNC survivors in the Irish population following completion of their primary treatment when compared with data from the NCRI database. Furthermore, to our knowledge, this is the first study to investigate the relationship between key self-management behaviours and QoL and FoR in HNC survivors and the first to investigate the relationship between self-management behaviours and FoR in cancer more generally; our findings clearly demonstrate the positive association between key self-management behaviours and these important outcomes.

\section{2 | Clinical implications}

The current findings suggest that developing effective ways of supporting self-management behaviours like active problem solving and positive self-talk ${ }^{27,39}$ may lead to increases in QoL and decreases in FoR amongst HNC survivors following treatment. The findings also have important implications for the design and implementation of supported self-management interventions for HNC survivors. Adjustment-focused self-management interventions supporting the acquisition of specific self-management skills ${ }^{36}$ may lead to positive effects in relation to QoL and FoR for HNC survivors. Furthermore, self-management intervention components related to self-monitoring may need to be specifically tailored to consider the potential for maladaptive self-monitoring amongst HNC survivors (and, potentially, amongst other cancer survivors). Health professionals should also be cognisant of the potential negative impact of this behaviour when advising HNC survivors on recognising and managing the signs and symptoms of recurrence and the development of specific screening tools to capture maladaptive hypervigilance may be useful for healthcare professionals to use in the clinical setting. Similarly, routine screening for maladaptive FoR (eg, using the Patient Concerns Inventory ${ }^{40}$ ) may help to identify those who need to be targeted with structured supported self-management programmes.

\section{ACKNOWLEDGEMENT}

This publication is independent research partly funded by the Irish Cancer Society and the Health Research Board in Ireland under Grant No: SRP13GAL, MRCG/2013/11.

\section{CONFLICTS OF INTEREST}

There is no conflict of interest to report.

\section{ORCID}

Simon Dunne (D) https://orcid.org/0000-0003-3655-7647

\section{REFERENCES}

1. Hoffman MA, Lent RW, Raque-Bogdan TL. A social cognitive perspective on coping with cancer: theory, research, and intervention. Counseling Psychol. 2013;41(2):240-267.

2. Foster C, Breckons M, Cotterell $\mathrm{P}$, et al. Cancer survivors' self-efficacy to self-manage in the year following primary treatment. J Cancer Surviv. 2015;9(1):11-19

3. McCorkle R, Ercolano E, Lazenby M, et al. Self-management: enabling and empowering patients living with cancer as a chronic illness. CA Cancer J Clin. 2011;61:50-62.

4. Davies N, Batehup L. Self-Management Support for Cancer Survivors: Guidance for Developing Interventions: An Update of the Evidence. London: Macmillan Group; 2010.

5. Paterson C, Rattray J, Lauder W. Identifying the self-management behaviours performed by prostate cancer survivors: a systematic review of the evidence. J Res Nurs. 2015;20:96-111.

6. Foster C, Fenlon D. Recovery and self-management support following primary cancer treatment. Br J Cancer. 2011;105:S21-S28.

7. Dingley $C$, Roux $G$. The role of inner strength in quality of life and selfmanagement in women survivors of cancer. Res Nurs Health. 2014:37:32-41.

8. Shneerson C, Taskila T, Holder R, et al. Patterns of self-management practices undertaken by cancer survivors: variations in demographic factors. Eur J Cancer Care. 2015;24(5):683-694.

9. Howell D, Harth T, Brown J, Bennett C, Boyko S. Self-management education interventions for patients with cancer: a systematic review. Support Care Cancer. 2017;25(4):1323-1355.

10. Howren MB, Christensen AJ, Karnell LH, Funk GF. Psychological factors associated with head and neck cancer treatment and survivorship: evidence and opportunities for behavioural medicine. J Consult Clin Psychol. 2013;81:299-317.

11. Djan R, Pennington A. A systematic review of questionnaires to measure the impact of appearance on quality of life for head and neck cancer patients. J Plast Reconstr Aes. 2013;66:647-659.

12. Dunne S, Mooney O, Coffey L, et al. Psychological variables associated with quality of life following primary treatment for head and neck cancer: a systematic review of the literature from 2004 to 2015 Psychooncology. 2016;26(2):149-160.

13. Ghazali N, Cadwallader E, Lowe D, Humphris G, Ozakinci G, Rogers $\mathrm{SN}$. Fear of recurrence among head and neck cancer survivors: longitudinal trends. Psychooncology. 2013;22(4):807-813.

14. Rogers SN, Audisio RA, Lowe D. Do the elderly raise different issues when using the Patient Concerns Inventory in routine head and neck cancer follow-up clinics? Eur J Cancer Care (Engl). 2015;24(2): 189-197.

15. Dunne S, Mooney O, Coffey L, et al. Self-management strategies used by head and neck cancer survivors following completion of primary treatment: a directed content analysis. Psychooncology. 2017;26(12): 2194-2200.

16. Dunne S, Coffey L, Sharp L, et al. Barriers to active self-management following treatment for head and neck cancer: survivors' perspectives. Psychooncology. 2018;27(10):2382-2388.

17. Dunne S, Coffey L, Sharp L, et al. Integrating self-management into daily life following primary treatment: head and neck cancer survivors' perspectives. J Cancer Surviv. 2018; Epub 2018 Dec 08. https://doi. org/10.1007/s11764-018-0726-4;1-13. 
18. Osborne RH, Elsworth GR, Whitfield K. The Health Education Impact Questionnaire (heiQ): an outcomes and evaluation measure for patient education and self-management interventions for people with chronic conditions. Patient Educ Couns. 2007;66(2):192-201.

19. Packer TL, Boldy D, Ghahari S, Melling L, Parsons R, Osborne RH. Self-management programs conducted within a practice setting: who participates, who benefits and what can be learned? Patient Educ Couns. 2012;87(1):93-100.

20. Heckel L, Fennell KM, Reynolds J, et al. Efficacy of a telephone outcall program to reduce caregiver burden among caregivers of cancer patients [PROTECT]: a randomised controlled trial. BMC Cancer. 2018;18(1):59.

21. Cella DF, Tulsky DS, Gray G, et al. The Functional Assessment of Cancer Therapy scale: development and validation of the general measure. J Clin Oncol. 1993;11(3):570-579.

22. List MA, D'Antonio LL, Cella DF, et al. The Performance Status Scale for Head and Neck Cancer Patients and the Functional Assessment of Cancer Therapy-Head and Neck Scale: a study of utility and validity. Cancer. 1996;77(11):2294-2301.

23. Greenberg DB, Kornblith AB, Herndon JE, et al. Quality of life for adult leukemia survivors treated on clinical trials of Cancer and Leukemia Group B during the period 1971-1988: predictors for later psychologic distress. Cancer. 1997;80(10):1936-1944.

24. Thewes B, Butow P, Zachariae R, Christensen S, Simard S, Gotay C. Fear of cancer recurrence: a systematic literature review of selfreport measures. Psychooncology. 2012;21(6):571-587.

25. Bush K, Kivlahan DR, McDonell MB, Fihn SD, Bradley KA. The AUDIT alcohol consumption questions (AUDIT-C): an effective brief screening test for problem drinking. Ambulatory Care Quality Improvement Project (ACQUIP). Alcohol Use Disorders Identification Test. Arch Intern Med. 1998;158(16):1789-1795.

26. Harding S, Sanipour F, Moss T. Existence of benefit finding and posttraumatic growth in people treated for head and neck cancer: a systematic review. PeerJ. 2014;2:e256. https://doi.org/10.7717/ peerj.256

27. Kolokotroni P, Anagnostopoulos F, Hantzi A. The role of optimism, social constraints, coping, and cognitive processing in psychosocial adjustment among breast cancer survivors. J Clin Psychol Med Settings. 2018; Epub Feb 21. https://doi.org/10.1007/s10880-018-9555$\mathrm{x} ; 25(4): 452-462$.

28. Yang YL, Li MY, Liu L, Wang L. Perceived stress and its associated demographic-clinical characteristics and positive expectations among Chinese cervical, kidney, and bladder cancer patients. Support Care Cancer. 2018;26(7):2303-2312.

29. Boland L, Bennett K, Connolly D. Self-management interventions for cancer survivors: a systematic review. Support Care Cancer. 2018;26(5):1585-1595.

30. Cnossen IC. Self-management supporting rehabilitation in head and neck cancer patients. Unpublished thesis. Amsterdam: Vrije Universiteit; 2016.
31. Fardell JE, Thewes B, Turner J, et al. Fear of cancer recurrence: a theoretical review and novel cognitive processing formulation. $J$ Cancer Surviv. 2016;10(4):663-673.

32. Reich M, Leemans CR, Vermorken JB, et al. Best practices in the management of the psycho-oncologic aspects of head and neck cancer patients: recommendations from the European Head and Neck Cancer Society Make Sense Campaign. Ann Oncol. 2014;25(11):2115-2124.

33. Stout RA, Beckman NJ, Shelby SR, Labott SM. Psychological issues in head and neck cancer. In: Radosevich JA, ed. Head \& Neck Cancer: Current Perspectives, Advances, and Challenges. New York: Springer; 2013:751-800.

34. Lin YH, Kao CC, Wu SF, Hung SL, Yang HY, Tung HY. Risk factors of post-traumatic stress symptoms in patients with cancer. J Clin Nurs. 2017;26(19-20):3137-3143.

35. Civilotti C, Castelli L, Binaschi L, et al. Dissociative symptomatology in cancer patients. Front Psychol. 2015;6:118.

36. Coffey L, Mooney O, Dunne S, et al. Cancer survivors' perspectives on adjustment-focused self-management interventions: a qualitative meta-synthesis. J Cancer Surviv. 2016;10(6):1012-1034.

37. Schuler M, Musekamp G, Bengel J, Nolte S, Osborne RH, Faller H. Measurement invariance across chronic conditions: a systematic review and an empirical investigation of the Health Education Impact Questionnaire (heiQ ${ }^{\text {TM }}$ ). Health Qual Life Outcomes. 2014;23(12):56.

38. Dodd RH, Waller J, Marlow LA. Human Papillomavirus and head and neck cancer: psychosocial impact in patients and knowledge of the link-a systematic review. Clin Oncol $(R$ Coll Radiol). 2016;28(7):421-439.

39. Hamilton R, Miedema B, MacIntyre L, Easley J. Using a positive selftalk intervention to enhance coping skills in breast cancer survivors: lessons from a community-based group delivery model. Current Oncology. 2011;18(2):e46-e53.

40. Rogers SN, Thomson F, Lowe D. The Patient Concerns Inventory integrated as part of routine head and neck cancer follow-up consultations: frequency, case-mix, and items initiated by the patient. Ann R Coll Surg Engl. 2018;100(3):209-215.

\section{SUPPORTING INFORMATION}

Additional supporting information may be found online in the Supporting Information section at the end of the article.

How to cite this article: Dunne S, Coffey L, Sharp L, et al. Investigating the impact of self-management behaviours on quality of life and fear of recurrence in head and neck cancer survivors: A population-based survey. Psycho-Oncology. 2019; 28:742-749. https://doi.org/10.1002/pon.5010 